Article

\title{
The Best of Both Worlds? Online Ties and the Alternating Use of Social Network Sites in the Context of Migration
}

\author{
Jens F. Binder ${ }^{1, *}$ and Alistair G. Sutcliffe ${ }^{2}$ \\ 1 School of Social Sciences, Nottingham Trent University, Burton Street, Nottingham NG1 4BU, UK \\ 2 Manchester Business School, University of Manchester, Booth Street West, Manchester M15 6PB, \\ UK; E-Mail: alistair.sutcliffe@mbs.ac.uk
}

* Author to whom correspondence should be addressed; E-Mail: jens.binder@ntu.ac.uk; Tel.: +44-115-8482416.

External Editors: Sonja Utz and Nicole Muscanell

Received: 3 June 2014; in revised form: 29 July 2014 / Accepted: 4 December 2014 /

Published: 11 December 2014

\begin{abstract}
While an ever-growing body of research is concerned with user behavior on individual social network sites (SNSs) — mostly Facebook—studies addressing an alternating use of two or more SNS are rare. Here, we investigate the relationship between alternating SNS use and social capital in the context of migration. Alternating SNS use avoids some of the problems associated with large networks located on one site; in particular the management of different social or cultural spheres. Not only does this strategy hold potential for increased social capital, it also provides a particular incentive for migrants faced with the challenge of staying in touch with back home and managing a new social environment. Two survey studies are presented that focus on the relationship between alternating SNS use and online ties in a migrant context involving Indian nationals. Study 1 looked at migration within India, whereas Study 2 compared international with domestic SNS users. In both studies, alternating SNS use added to the prediction of online network size and accounted for differences in network size found for migrant and non-migrant users. Differences were due to the number of peripheral ties, rather than core ties. Findings suggest that alternating SNS use may constitute a compensatory strategy that helps to overcome lower levels of socializing represented through a single SNS.
\end{abstract}

Keywords: Orkut; migration; alternating SNS use; online network; online network size 


\section{Introduction}

In this work, we investigate links between an individual's use of more than one social network site (SNS) [1] and online social contacts in the context of migration. While the use of a range of social media channels for network maintenance is well documented in past research $[2,3]$, the detailed usage pattern for multiple SNSs has not received similar attention. In the following, such usage is referred to as alternating use, without implying any particular temporal sequence. The alternating use of two or more SNSs, we argue, facilitates the accumulation of social capital and constitutes a user strategy of particular relevance in the context of migration and fragmented social capital. In the present paper, a migrant is defined as a person who has left a home location by crossing substantial geographical boundaries, usually for work/study reasons, and still wishes to maintain ties with this home location and with known others who have also left. Information technology generally has helped migrants to stay connected to their geographical origins [4-8], but to date little is known about SNS use and migration. Recent work on transnational Human-Computer Interaction [9] and cross-cultural SNS use [10] has highlighted the relevance of long-distance technologies for particular user groups. SNS have famously been shown to enable the accumulation and consolidation of social capital [11,12], in particular for users moving to a different location. This bridging of geographical distance carries enormous potential for improving the quality of social life for migrants, but may also pose additional challenges [8].

Migrants often not only switch between locations, but also between cultures. This has implications in terms of different online user groups migrants want to stay in touch with and the different user practices and preferred online services that these groups display. Put differently, the intercultural context of migration provides a particular driver for becoming continuously active on more than one SNS at the same time. SNS use among migrants therefore can indicate both the need to bridge cultural boundaries and to keep up with changing user practices among peers [5,8]. Alternating SNS use should help to explain differences between migrants and non-migrants in terms of online socializing and accumulated social capital. In the present work, this is captured by focusing on alternating Orkut and Facebook use among Indian nationals within India and in the West.

While there has been little doubt that SNSs are well-suited to optimize online socializing at different levels, for example, friendships, professional ties, special interest groups, considerable debate has focused on the actual social gains to be expected from such services [13]. An intermediate conclusion from research on online social capital suggests positive gains, albeit less so in terms of increasing the intensity of relationships, more so in terms of quantity of relationships and the upkeep of existing relationships that would otherwise fall in disuse $[11,12,14]$. One of the most important top-level outcomes in these studies is network size; and with few exceptions that concern user credibility [15] or addictive behaviors [16], larger online networks are generally taken as positive outcomes of SNS use in the literature [13,17-19]. In this work, we focus on overall network size and number of core ties as main indicators of social capital. These indicators map onto the distinction between weak and strong ties [20] and bridging and bonding capital [11,14], respectively. While strong ties, and bonding capital, are seen as a vital sources of emotional and instrumental support [21], the ability of SNSs to increase such sources has been questioned from early on [22]. In contrast, increases in weak ties appear as a particular strength of SNSs as reflected in the frequently observed inflation of online network size [23]. Weak ties accrue value by allowing users to reach beyond the confines of their well-known friendship 
and family clique for new information and specialist advice [20]. However, while weak ties come with little maintenance cost, their unrestricted increase within one particular service may generate unforeseen challenges.

Two fundamentally different sets of strategies are open to SNS users that are likely to secure existing online social ties and to increase the overall number of such ties: strategies that work within a particular SNS and strategies that work across sites. Within a particular site, the simplest user strategy would be to increase overall activity levels by increasing the frequency of use $[24,25]$ as well as the intensity of it, with the aim of increasing social outreach online. Intensity may be about overall importance a user assigns to an SNS [11], or it may extend to social versus non-social uses of particular site features [12,14], for example photo-sharing [26]. It has been suggested, though, that strategies operating within sites can easily increase the likelihood of social tension due to the emergence of conflicting social spheres [27] and the neglected use of privacy settings in the face of unmeditated broadcasting of information [28]. In other words, larger networks may be heading towards a stage at which they become socially unmanageable, in particular as they become more socially diverse and include different forms of social capital.

Managing different social spheres across sites, through the use of different SNSs, offers one way out of this dilemma. While there is an abundance of anecdotal evidence for user engagement with several social media, and in particular SNS, at the same time, little is known about the implications for online network size. In fact, existing studies rarely touch upon alternating SNS in general. For example, in their seminal overview of SNS, Boyd and Ellison [1] describe switching from one SNS to the other rather than the alternating maintenance of two or more profiles by one user; neither has this been a feature of psychologically relevant research in the few years since the rapid expansion of Facebook [13]. Some studies that have provided general surveys of SNS use within well-defined communities [29,30] strongly suggest alternating use of SNS without addressing this topic directly in the analyses. In sum, we would expect that alternating SNS use opens up possibilities for further growth of the overall number of online contacts. In terms of the types of capital identified in the literature [11,14], alternating SNS use should be instrumental in the accumulation of weak ties, rather than strong ties, similar to other types of SNS uses investigates so far.

Migrants constitute a special user group in the context of alternating SNS use due to the constant bridging of cultures implied by their circumstances [31]. Where this bridging takes place online, the task is essentially one of managing different social spheres, as outlined above. In addition, migrants have to accommodate user practices from different cultures that work against the consolidation of online networks on one single SNS. It would not be feasible, for example, to persuade online contacts to switch to an SNS that, to them, is not culturally established. Depending on the intercultural context, users may have to use more than one particular SNS and maintain several online presences in order to stay in touch with back home and to embrace a new social environment. The management of such hybrid communities has recently been identified as one of the main challenges in a transnational perspective on Human-Computer Interaction [9], and early evidence suggests that users are sensitive to the cultural context suggested by a particular SNS [10,32]. We extend this perspective by proposing that alternating SNS use may be a necessity as well as a given for migrants.

In terms of acculturation theory [31], migrants should reap most psychological benefits from a strategy in which they manage to integrate both a cultural context back home and the present context in 
which they reside. Similarly, social support networks drawing on contacts back home and in new surroundings are both important for immigrant well-being [33]. As Chen [34] has shown for general Internet use, migrants draw particular benefit from a balanced use of websites from home country and country of residence. A similarly balanced use of ICTs among migrants has been described by Burrell and Anderson [5], whereas Komito [8] has raised concerns over an imbalanced SNS use that could bind migrants more to their home community and as such work against societal integration see also [35]. These findings lend further support to the idea that migrants can have the best of both, or more, worlds through careful management of contact across sites. In sum, we would expect these advantages, combined with the continuous, every-day experience of bridging cultures, to lead migrants toward alternating SNS use.

The bridging of cultures is one factor that should drive migrants towards alternating SNS use. At the same time, predicting the actual levels of online social capital that migrants hold is by no means straightforward. Migrants may be disadvantaged compared to others in their current place of residence, as individuals driven from home through economic factors and in need of cultural re-adjustment [31]. Migrants may also be regarded as advantaged compared to others in their place of origin, as technology-savvy individuals who have the support and resources to pursue careers and studies for the highly skilled and qualified. A similar ambiguous status has been ascribed to international students who, in spite of a high standing and educational level, experience some of the same acculturative issues that disadvantaged groups do [36,37]. We therefore refrained from speculating on the actual levels of capital for migrant and non-migrant groups and focused on the compensatory function of alternating SNS use instead. If alternating SNS use is effective in increasing the number of weak ties, it should account for differences in this type of social capital between migrant and non-migrant users.

What technologies posing as SNSs are available to migrant users? Globally, strong geographical variations in SNS distribution have been documented [1]. While Facebook is by now known in virtually any country with online access, there are a number of country-specific SNSs that are not much known outside their user population such as Cyworld in South Korea [38] and RenRen in China [10]. For a decade, Orkut was successful in maintaining a vast user base in Brazil and in India, but had insignificant impact in the US and Europe [1,32]. While crossing boundaries between East and West, such culturally accepted and culturally shared SNSs at the country-level are probably best suited for alternating SNS use and should allow for staying in touch with a growing, diversifying social network and minimizing intercultural blunders [28,39].

\section{Hypotheses}

Based on the theoretical considerations so far, several hypotheses can be postulated. Given the likely benefits of alternating SNS use specifically for migrant groups, we would expect these users to engage more in this practice:

H1: Migrant users engage more in alternating SNS use than non-migrant users.

More generally, alternating SNS use opens up more possibilities for network expansion by avoiding increased maintenance demands that arise from online networks managed through one site:

H2: Alternating SNS use is positively associated with the overall number of online contacts. 
To the extent that migrants take on the acculturative challenges that come with global mobility through different forms of online socializing, alternating SNS use should account for differences between migrant and non-migrant user groups in terms of online social capital:

H3: Alternating SNS use will compensate for differences in network size between migrant and non-migrant users.

Finally, given previous theorizing and findings, we would expect alternating SNS use to be related to social capital that stems from weak, rather than strong, ties:

H4: Alternating SNS use is positively associated with the number of weak ties, rather than strong ties.

The present work summarizes two studies on Orkut and Facebook use among Indian nationals within India and in the West, conducted at a time when both services were common among users in India (2008/2009). We investigated scenarios in which Indian SNS users had moved a long distance from a technologically less advanced to a more advanced region for career or education purposes, within India (Study 1) and internationally (Study 2). Scenarios were chosen to capture users who had pre-existing contacts on Orkut and added online socializing activities when they adopted Facebook. Although both scenarios also differed markedly from each other, we expected that the basic function of alternating SNS use would remain the same: to allow for the accumulation of more online contacts. In both studies, following our hypotheses, our approach was to compare alternating SNS among migrant and non-migrant users on accumulated online contacts and strong ties. In particular, we tested whether alternating SNS use would: (a) serve as a useful additional predictor of online network size, over and above intensity of SNS use as well as user age and gender; and (b) account for differences indicated by migrant status.

\section{Study 1}

In Study 1, we compared users from two very different regions within India: a distinct cultural area with comparatively weak infrastructure in the north east of the country (the states of Mizoram, Manipur and Nagaland) and major urban centers in the south (Bangalore and Hyderabad) with highly developed infrastructure and strong IT communities. The north eastern states rank among the poorest in the Indian Union and belong to a geographical area that is almost cut off from the main landmass. Within India, the inhabitants of this area are typically seen as quite distinct from the mainlanders in terms of culture, language, and even genetic makeup. Travel distances over land to the southern urbanities are in the region of $3000 \mathrm{~km}$.

\subsection{Method}

We relied on sampling participants through one central network owner, or ego, to ensure an overall homogeneity of respondents due to homophily [40,41]. Participants were recruited from personal online networks maintained by an Indian national and responded to an online survey that was accessible for four weeks. 
Of the 118 respondents, 51\% were male and 49\% female with the majority (74\%) being in the age band of 21 to 25 years. All respondents were Indian nationals and residents, either employed in professional positions or as students at colleges and universities. The majority had origins in the north eastern region (71\%) whereas the others originated from further south. The sample included a group of migrants (56\%), all of whom had moved from the north east to the south. Further, 25\% reported to be Orkut only users whereas 30\% used two SNSs, $27 \%$ used three, $15 \%$ used four, and $2.5 \%$ used five. The vast majority, 92\%, had registered first on Orkut and reported to use Orkut as their main site. Table 1 shows information on SNS use by migrant status.

Table 1. Sample Composition, Study 1, by migrant status and number of SNS in use.

\begin{tabular}{cccccc}
\hline \multirow{2}{*}{ Status } & \multicolumn{5}{c}{ SNS in Use } \\
\cline { 2 - 6 } & $\mathbf{1}$ (Orkut) & $\mathbf{2}$ & $\mathbf{3}$ & $\mathbf{4}$ & $\mathbf{5}$ \\
\hline Non-migrant & 10 & 12 & 16 & 12 & 2 \\
Migrant (from north eastern India) & 20 & 23 & 16 & 6 & 1 \\
\hline
\end{tabular}

Along with participants' age and gender, the following measures were obtained. Number of SNS in use. Respondents were asked to select all SNS they were using in addition to Orkut from a list containing six sites (Facebook, Bebo, Friendster, Hi5, LinkedIn, MySpace). Sites were chosen on the basis of their estimated popularity within India at the time of data collection. In addition, participants were asked to enter any other SNS in free format. The number of sites ticked and named was used as a measure of alternating SNS use.

Orkut network size. Given that Orkut was the main site in use and that overlap among multiple SNS profiles is difficult to control, we chose the number of Orkut contacts as the main outcome measure for this study. We would still expect alternating SNS use to be related to Orkut contacts since user activity on other SNS can serve to consolidate and complement activity on Orkut. For example, contacts found through more specialized sites-dealing, for example, in professional or gaming issues - could be persuaded to join the main Orkut profile. Conversely, discussions on Orkut could accompany, or spark, activities on other sites. As is typically found, the distribution was heavily skewed and long-tailed. It was therefore decided to use a logarithmic transformation of this measure.

Intensity of Orkut use. A single item asked for the amount of time spent on Orkut daily, with the following answering options: $<1$ h, 1 h, 2 h, 3 h, 4 h, $>4$ h. Options were coded as one to six $(M=2.19$, $\mathrm{SD}=1.17$ ), with higher numbers indicating longer daily Orkut use.

Tie strength. For a more detailed account of online network composition, participants were asked to estimate the number of online contacts at four levels of closeness: "best friends/very strong relation", “friends/normal relation”, “acquaintances/weak relation” and "strangers/haven’t met in real life”. For each level, a six point scale was used with the following options: 1-10, 11-20, 21-40, 41-80, 81-150, $151+$. Options were coded as one to six, with higher numbers indicating more ties. Means for best friends, friends, acquaintances and strangers were $\mathrm{M}=1.43$ ( $\mathrm{SD}=0.92), \mathrm{M}=3.71$ ( $\mathrm{SD}=1.27$ ), $\mathrm{M}=3.67$ (SD = 1.91) and $\mathrm{M}=2.33$ (SD = 1.37), respectively.

Migrant status. Respondents also indicated their region of origin and region of residence. Migrants were classified as those with different places of origin and residence. Non-migrant users were coded as 0 and migrant users as 1. 


\subsection{Results}

Migrant users $(M=2.17, S D=1.02)$ listed fewer SNS than non-migrant users $(M=2.69, S D=1.14)$ : $t(116)=2.64, p=0.01$. They also showed smaller online networks than non-migrant users (2.17 vs. 2.32, $t(102)=2.43, p=0.02)$. While no significant differences between user groups were found for best friends/very close relationships and friends in general terms, migrant users reported smaller numbers for both acquaintances (3.22 vs. 4.30, $t(91)=2.83, p<0.01)$ and offline strangers (2.08 vs. 2.73, $t(89)=2.31, p=0.02)$. Mean scores are reported in Table 2.

Table 2. Mean scores and standard deviations (in parentheses) for non-migrant and migrant users.

\begin{tabular}{cccc}
\hline \multirow{2}{*}{ Network Indicator } & \multicolumn{2}{c}{ User Status } & \multirow{2}{*}{$\boldsymbol{p}$} \\
\cline { 2 - 3 } & Non-Migrant & Migrant & \\
\hline Network size & $249.02(135.10)$ & $189.09(126.17)$ & 0.02 \\
Logged network size & $2.32(0.28)$ & $2.17(0.32)$ & 0.02 \\
Best friends/very strong relation & $1.41(0.96)$ & $1.43(0.79)$ & $\mathrm{ns}$ \\
Friends in general & $3.94(1.13)$ & $3.56(1.34)$ & $\mathrm{ns}$ \\
Acquaintances & $4.30(1.79)$ & $3.22(1.85)$ & $>0.01$ \\
Offline strangers (not met face-to-face) & $2.73(1.27)$ & $2.08(1.40)$ & 0.02 \\
\hline
\end{tabular}

In order to test whether migrant users would engage more in alternating SNS use (H1), a $t$-test was conducted on the number of SNS as reported in Table 1. Contrary to expectations, non-migrant users $(\mathrm{M}=2.73, \mathrm{SD}=1.13)$ reported using more SNS than migrant users $(\mathrm{M}=2.15, \mathrm{SD}=1.02, t(116)=2.90$, $p<0.01)$.

To investigate associations between alternating SNS use and network size, thus testing $\mathrm{H} 2$, we carried out hierarchical linear regression analyses. In a first step, logged network size was regressed on age, gender (coded as 0 for males and 1 for females), and intensity of Orkut use as control variables. In a second and third step, migrant status and the number of SNSs in use were added. Intercorrelations are reported in Table 3; regression results are summarized in Table 4. Being a migrant was significantly related to smaller network size in Step $2(\beta=-0.19, p<0.05)$. This association was weakened and not significant in Step 3 ( $\beta=-0.11$, ns) where the number of SNS turned out to be a positive predictor of network size $(\beta=0.34, p<0.01)$, in line with H2. Changes from Step 1 to Step 2 were significant $(F(1,98)=5.64, p=0.02)$, as were the changes from Step 2 to Step $3(F(1,97)=12.00, p<0.01)$. Thus, supporting H3, alternating SNS use accounted for the lower network sizes found for migrants, rendering the difference between migrant and non-migrant users insignificant.

We proceeded in the same way for different levels of closeness in the network. Migrant status and alternating SNS use did not play any role for the two closest types of contacts. For acquaintances and very weak relations, however, the same pattern was found as for the overall network, confirming H4. Migrant status negatively predicted weak relations in Step $2(\beta=-0.26, p=0.01)$, but was not significant in Step $3(\beta=-0.19$, ns) where the number of SNS $(\beta=0.25, p=0.02)$ showed a positive association. Similar findings were obtained for offline strangers: migrant status was significant in Step 2 ( $\beta=-0.21, p<0.05)$, but not significant in Step $3(\beta=-0.15$, ns) where the number of SNS was the only significant predictor $(\beta=0.24, p<0.03$ ). In sum, while closer relationships were unrelated to 
migrant status and alternating SNS use, the pattern for less close relationships was the same as for the overall network.

Table 3. Intercorrelations for predictors and logged Orkut network size. Upper half matrix refers to non-migrant users; lower half matrix to migrant users.

\begin{tabular}{|c|c|c|c|c|c|c|}
\hline Predictor & Predictor Number & 1 & 2 & 3 & 4 & 5 \\
\hline Age & 1 & - & -0.09 & -0.07 & 0.06 & -0.10 \\
\hline Gender & 2 & 0.04 & - & -0.20 & $-0.32 *$ & -0.08 \\
\hline Intensity & 3 & -0.08 & -0.07 & - & 0.13 & 0.27 \\
\hline Number of SNS & 4 & 0.16 & 0.03 & 0.17 & - & $0.39 * *$ \\
\hline Network size & 5 & -0.02 & $0.29 *$ & $0.29 *$ & 0.23 & - \\
\hline
\end{tabular}

Table 4. Summary of regression results for predicting Orkut network size from migrant status and simultaneous SNS use, controlling for age, gender and intensity of use.

\begin{tabular}{|c|c|c|c|c|}
\hline Predictor & $\bar{b}$ & $\mathrm{SE}$ & $\beta$ & $p$ \\
\hline \multicolumn{5}{|c|}{ Step $1\left(R^{2}=0.13\right)$} \\
\hline Gender & 0.11 & 0.06 & 0.17 & ns \\
\hline Age & -0.01 & 0.05 & -0.01 & ns \\
\hline Intensity of use & 0.09 & 0.02 & 0.36 & $* * *$ \\
\hline \multicolumn{5}{|c|}{ Step $2\left(R^{2}=0.17\right)$} \\
\hline Gender & 0.11 & 0.06 & 0.18 & ns \\
\hline Age & -0.01 & 0.05 & -0.02 & ns \\
\hline Intensity of use & 0.08 & 0.02 & 0.32 & $* *$ \\
\hline Migrant status & -0.12 & 0.06 & -0.19 & $*$ \\
\hline \multicolumn{5}{|c|}{ Step $3\left(R^{2}=0.27\right)$} \\
\hline Gender & 0.15 & 0.06 & 0.25 & $* *$ \\
\hline Age & -0.03 & 0.05 & -0.06 & ns \\
\hline Intensity of use & 0.08 & 0.02 & 0.29 & $* *$ \\
\hline Migrant status & -0.07 & 0.06 & -0.11 & ns \\
\hline Number of SNS & 0.10 & 0.03 & 0.34 & $* *$ \\
\hline
\end{tabular}

$* p<0.05 ; * * p<0.01 ; * * * p<0.001$.

\subsection{Discussion}

Migrant users reported markedly smaller online networks when compared to non-migrant users, and they reported a lower number of SNSs in use. While this does not at first support our expectations that migrants would be particularly drawn to the adoption of more than one SNS, alternating SNS use did show a positive association with Orkut network size and accounted for differences in network size between migrant and non-migrant users. In other words, as migrants engaged with more SNSs, differences in network size became non-significant. These findings are not explained by age, gender or the intensity of Orkut use.

We see alternating SNS use as a compensatory strategy for migrants that helps them in consolidating online contacts and potentially overcoming acculturative challenges. This benefit seems restricted to 
less close relationships, which confirms earlier notions that SNS serve mainly the accumulation of weak ties [42]. A more detailed discussion of findings is to follow further down, in conjunction with the outcomes from Study 2.

\section{Study 2}

Study 1 focused on a specific migration scenario within India, and assessed online contacts for one main SNS only. The second study, as before, investigated SNS use among Indians who kept Orkut as their main site. Choosing a different intercultural context, however, international migrant user, residing in the US and the UK were compared to domestic non-migrant users. Further, Orkut-only users and dual users active on Orkut and Facebook were recruited. The popularity of these modes of SNS use was evident in Study 1 where both user groups accounted for almost 50\% of the entire sample. In addition, a selection of these user groups allowed us to estimate the total number of SNS contacts, as described below. In other respects, our approach was identical to that in Study 1.

\subsection{Method}

Participants were recruited through the personal online networks maintained by an Indian national and responded to an online survey that was accessible for four weeks. Of the 189 Indian respondents (34\% female, mean age $=24.63, \mathrm{SD}=3.91), 119(63 \%)$ were currently residing in India whereas 70 (37\%) lived in the US or the UK, 53\% were Orkut-only users, $47 \%$ used both Orkut and Facebook. The vast majority (94\%) had registered first on Orkut and reported to use Orkut as their main site. Table 5 shows information on SNS use by migrant status.

Table 5. Sample Composition, Study 2, by migrant status and SNS in use.

\begin{tabular}{ccc}
\hline \multirow{2}{*}{ Migrant Status } & \multicolumn{2}{c}{ SNS Use } \\
\cline { 2 - 3 } & Orkut & Dual \\
\hline Non-migrant & 74 & 45 \\
Migrant & 26 & 44 \\
\hline
\end{tabular}

Several steps were taken to improve the quality of measures in comparison to Study 1: we obtained number estimates for Orkut and Facebook contacts and the overlap between the two; we used previously validated measures for the number of close online ties; and we distinguished between frequency and duration of Orkut use rather than relying on a single-item measure of use intensity. Along with participants' age and gender, the following measures were used.

Alternating SNS use. Respondents were asked whether they used Facebook in addition to Orkut. For the analyses, Orkut-only users were coded as 0 and dual users as 1.

Migrant status. Respondents also indicated whether they resided in their home town, in a different place within India, or overseas (US and UK). For the analyses, non-migrant users (residing within India) were coded as 0 and migrant users as 1.

Online network size. Participants entered the number of Orkut contacts. For dual users, the number of Facebook contacts was asked for as was the number of contacts on both Orkut and Facebook. 
Online network size was defined as Orkut plus Facebook contacts minus the overlap. As in Study 1, the distribution was heavily skewed and long-tailed such that a logarithmic transformation was used.

Strong ties. Participants responded to two prompts to capture core emotional ties $[21,43,44]$ with regard to their online contacts: all the people whom they would ask for help in times of emotional trauma or financial distress and, in addition, all the people whose death would leave them personally devastated. Following previous studies, both numbers were summed up. This index was then subjected to a logarithmic transformation.

Frequency of Orkut use. This was assessed with the following response options: less than once per month, once per month, twice per month, 1-2 times a week, 3-4 times a week, almost daily. Options were coded as one to six, with higher numbers indicating higher frequency of use.

Duration of Orkut use. Participants indicated how long, on average, their Orkut sessions lasted, with four options: less than $15 \mathrm{~min}, 1-2 \mathrm{~h}, 3-4 \mathrm{~h}$, more than $4 \mathrm{~h}$. These were coded as one to four with higher numbers indicating longer duration.

\subsection{Results}

Migrant users $(\mathrm{M}=2.17, \mathrm{SD}=0.30)$ showed larger Orkut networks than non-migrant users $(\mathrm{M}=2.04, \mathrm{SD}=0.39): t(137)=2.12, p<0.05$. The same held for the combined network of Orkut and Facebook contacts (2.26 vs. 2.09, $t(137)=2.62, p=0.01)$. As in Study 1 , differences were insignificant for closer contacts. Mean scores are summarized in Table 6.

Table 6. Mean scores and standard deviations (in parentheses) for non-migrant and migrant users.

\begin{tabular}{cccc}
\hline \multirow{2}{*}{ Network Indicator } & \multicolumn{2}{c}{ User Status } & \multirow{2}{*}{$\boldsymbol{p}$} \\
\cline { 2 - 3 } & Non-Migrant & Migrant & ns \\
\hline Orkut network size & $151.05(120.98)$ & $189.02(138.78)$ & 0.04 \\
Logged Orkut network & $2.04(0.39)$ & $2.17(0.30)$ & 0.02 \\
Overall network size & $168.64(131.85)$ & $226.74(155.64)$ & 0.01 \\
Logged overall network & $2.09(0.39)$ & $2.26(0.31)$ & $\mathrm{ns}$ \\
Close SNS ties & $35.43(35.42)$ & $36.76(22.53)$ & $\mathrm{ns}$ \\
Logged close ties & $1.36(0.43)$ & $1.46(0.36)$ & \\
\hline
\end{tabular}

In order to test whether migrant users would engage more in alternating SNS use (H1), a $\chi^{2}$-test was conducted on the frequencies as reported in Table 5. In line with expectations, migrant users showed a higher proportion of dual users than non-migrant users: $\chi^{2}=11.09, p<0.01$.

We then proceeded with the same regression approach as in Study 1 to investigate associations between alternating SNS use and network size. In the first step of a hierarchical linear regression, logged overall network size was regressed on age, gender, frequency and duration of Orkut use as control variables. In a second and third step, migrant status and dual SNS use were added as predictors. Intercorrelations are reported in Table 7; regression results are summarized in Table 8. 
Table 7. Intercorrelations for predictors and logged Orkut network size and logged overall network size. Upper half matrix refers to non-migrant users; lower half matrix to migrant users.

\begin{tabular}{ccccccccc}
\hline Predictor & Predictor Number & $\mathbf{1}$ & $\mathbf{2}$ & $\mathbf{3}$ & $\mathbf{4}$ & $\mathbf{5}$ & $\mathbf{6}$ & $\mathbf{7}$ \\
\hline Age & 1 & - & $-0.25^{* *}$ & $-0.26^{*}$ & -0.16 & -0.12 & -0.19 & -0.15 \\
Gender & 2 & 0.17 & - & 0.12 & $0.22 *$ & -0.03 & 0.01 & -0.15 \\
Frequency & 3 & 0.03 & 0.24 & - & 0.15 & 0.04 & $0.39 * * *$ & $0.26 * *$ \\
Time & 4 & $0.41^{* * *}$ & 0.12 & 0.18 & - & 0.16 & 0.09 & 0.07 \\
Alternating SNS use & 5 & $-0.29 *$ & -0.12 & 0.11 & -0.16 & - & $0.25 *$ & $0.41 * * *$ \\
Orkut network & 6 & 0.06 & -0.01 & 0.23 & $0.33 *$ & 0.18 & - & $0.91 * * *$ \\
Overall network & 7 & -0.03 & -0.12 & 0.21 & 0.28 & $0.39 * *$ & $0.94 * * *$ & - \\
\hline
\end{tabular}

$* p<0.05 ; * * p<0.01 ; * * * p<0.001$.

Table 8. Summary of regression results for predicting online network characteristics from migrant status and dual SNS use, controlling for age, gender, frequency of Orkut use and duration of Orkut sessions.

\begin{tabular}{ccccccc}
\hline Predictor & \multicolumn{3}{c}{ Orkut Network } & \multicolumn{3}{c}{ Overall Network } \\
\hline Step 1 & $\boldsymbol{b}$ & SE & $\boldsymbol{\beta}$ & $\boldsymbol{b}$ & SE & $\boldsymbol{\beta}$ \\
\hline Age & -0.01 & 0.01 & -0.07 & -0.01 & 0.01 & -0.07 \\
Gender & -0.01 & 0.07 & -0.13 & -0.12 & 0.07 & -0.15 \\
Frequency & 0.10 & 0.02 & $0.38^{* * *}$ & 0.07 & 0.02 & $0.28 * *$ \\
Time & 0.06 & 0.04 & 0.11 & 0.06 & 0.04 & 0.11 \\
\hline Step 2 & \multicolumn{3}{c}{$R^{2}=0.20$} & & & $R^{2}=0.15$ \\
\hline Age & -0.01 & 0.01 & -0.10 & -0.01 & 0.01 & -12 \\
Gender & -0.09 & 0.07 & -0.12 & -0.11 & 0.07 & -0.14 \\
Frequency & 0.09 & 0.02 & $0.35 * * *$ & 0.06 & 0.02 & $0.23 *$ \\
Time & 0.06 & 0.04 & 0.11 & 0.06 & 0.04 & 0.11 \\
Migrant status & 0.09 & 0.07 & 0.12 & 0.14 & 0.07 & $0.18 *$ \\
\hline Step 3 & \multicolumn{3}{c}{$R^{2}=0.23$} & & & $R^{2}=0.27$ \\
\hline Age & -0.01 & 0.01 & -0.08 & -0.01 & 0.01 & -0.07 \\
Gender & -0.06 & 0.07 & -0.07 & -0.04 & 0.06 & -0.05 \\
Frequency & 0.09 & 0.02 & $0.35 * * *$ & 0.06 & 0.02 & $0.23 * *$ \\
Time & 0.05 & 0.04 & 0.09 & 0.03 & 0.04 & 0.07 \\
Migrant status & 0.06 & 0.07 & 0.08 & 0.08 & 0.07 & 0.10 \\
Dual SNS use & 0.13 & 0.06 & $0.18 *$ & 0.27 & 0.06 & $0.37 * * *$ \\
\hline
\end{tabular}

$* p<0.05 ; * * p<0.01 ; * * * p<0.001$.

Being a migrant was significantly related to larger network size in Step 2 ( $\beta=0.18, p<0.05$ ). However, this association was weakened and not significant in Step $3(\beta=0.10$, ns) where dual SNS use turned out to be a positive predictor of network size $(\beta=0.37, p<0.01)$, in line with H2. Thus, supporting H3, dual SNS use accounted for the larger network sizes found for migrant users. Changes from Step 1 to Step 2 were significant $(F(1,133)=4.17, p=0.04)$, as were the changes from Step 2 to Step $3(F(1,132)=21.76, p<0.01)$. We repeated the analysis using logged Orkut network size as the outcome variable. Although dual SNS use remained a positive predictor of Orkut network size $(\beta=0.18$, 
$p<0.05)$ in Step 3, migrant status was no longer significant at any step. While changes from Step 1 to Step 2 were not significant $(F(1,133)=1.84$, ns $)$, changes from Step 2 to Step 3 were $(F(1,132)=5.42$, $p=0.02)$. For number of close relationships, no significant predictors emerged at any step, indicating that associations between alternating SNS use and network size were driven by weak ties, confirming H4.

\subsection{Discussion}

Similar to Study 1, dual SNS use accounted for differences between migrant and non-migrant users in overall online network size. As before, this is in line with an interpretation of alternating SNS use as a compensatory strategy. In addition, migrants showed a greater likelihood to use both SNSs. This is not to say that the domestic, non-migrant group was confined to Orkut-only use and international migrant users routinely used two SNS. Our analyses rather seem to indicate that dual use as a practice weighs more heavily on network size than differences associated with migration. Given the tremendous changes that come with migration for countless aspects of an individual's life, the overriding role this user practice plays is remarkable. As before, our findings were obtained while controlling for user age, gender, and activity levels on Orkut. Also consistent with Study 1, differences related to migrant status or alternating SNS use were not due to core ties in the network. While we did not measure weak ties in the network directly, their number is determined by overall network size minus number of core ties. Our findings therefore imply that any observed network growth is driven by weak ties.

Migrant users, contrary to Study 1, reported higher numbers of SNS contacts, both on Orkut and also for the combined Orkut and Facebook network. This may be down to a better resourced migrant group in the sample. It could be, for example, that more material and social resources are needed to move outside of India in comparison to a long-distance change in location within the country. Alternatively, there may have been differences in terms of the local support groups in both samples that our measures were not designed to detect. If migrant users in Study 1, for example, had a stronger local support group consisting of members of their home community than migrant users in Study 2, they would have had less reason to engage in alternating SNS use and in building larger networks. A closer investigation of local support structures, however, is beyond the scope of the present work.

\section{General Discussion and Conclusions}

The present work adds to the ever-growing research literature on SNSs by focusing on a particular site, Orkut, and a particular set of user groups, all Indian nationals, that both usually do not receive researchers' attention. Furthermore, its basic aim is to introduce the concept of alternating SNS use and its relationship with online network size in the context of migration. In the following, we will first examine the status of our hypotheses and then elaborate on some of the wider implications of our findings.

H1 stated that migrant users would engage more in alternating SNS use than non-migrant users. While this was supported in Study 2, in an international context, the exact opposite was found in Study 1, in a domestic context. This could point to a crucial distinction between user strategies that are normatively desirable and those that are actually observed. A user group is under no obligation to take up a practice, only because this would carry benefits specifically for this group. An analogy can be found in acculturation theory [31], where an integrative acculturation strategy, even though it signifies a range of positive outcomes, is by no means adopted by all members of an immigrant group. In addition, 
there might be differences in opportunities for face-to-face contact between the migrant groups in both studies. For example, domestic travel costs within India are far lower than international travel costs, which could have implications for the frequency of "home visits" and the perceived benefits derived from alternating SNS use.

H2 stated that alternating SNS use would be positively associated with online network size, an expectation that was supported in both studies. Although we find it plausible to assign a causal role to SNS use in this context, we are well aware of the fact that our data cannot directly address causal processes. An alternative account could be, for example, that network size drives further SNS use. Such a process would imply, however, that growing networks on one site lead to a partial transfer of the network to additional sites, with considerable time and effort required from the user. As outlined in the beginning, it is unlikely that a user could persuade existing ties to follow such a transfer. A more detailed empirical answer to this question may come from longitudinal studies that allow for the tracking of changes over time.

H3 stated that alternating SNS use would account for differences in network size between migrant and non-migrant users. This received support in both studies such that differences between user groups became non-significant once SNS use was controlled for. We therefore suggest that alternating SNS use constitutes, firstly, a user practice that is effective to bridge differences in social capital likely to be found in the context of migration; secondly, this practice can serve as an explicit strategy to compensate for inequalities in online social contacts. These considerations lead to the more general theme of dealing with geographical dispersal through Internet technology. Migrants carry the potential of large and diversified sets of social ties with them, but only with the advent of easily available Internet services has it become possible to pool this dispersed social capital in one (virtual) place and to turn dispersal into an advantage. Our work indicates that such a process does not happen automatically, but requires specific user behaviors.

H4 stated that alternating SNS use would be associated with weak, rather than strong, ties. As was shown in Study 1, effects were indeed sensitive to levels of closeness in a network, and no effects were obtained for best or moderate friends. This has implications for the type of social capital to expect. If the added use of more SNSs merely accelerates the accumulation of weak ties, SNS users, migrant or otherwise, are still well advised to build their bonding capital through other means.

Previous literature $[27,28]$ has suggested that conflicting social spheres can pose difficulties in the maintenance of growing online networks due to the increased likelihood of online clashes and tensions that run across such spheres. Alternating SNS use constitutes a user strategy that avoids these problems and opens up the possibility for unrestricted network growth by letting different SNSs act as naturally occurring segmentations of users' social capital. The present work extends the notion of social spheres by focusing on the intercultural context of migration. Cultural spheres may be seen as an instance of social spheres on a more global scale. Migrants, who are almost inevitably exposed to a variety of cultural spheres, are therefore a main target group for alternating SNS use. However, to the extent that technology assists more and more user groups in the bridging of long geographical distances, we can expect alternating SNS use to become a much more general user practice.

In our studies, it was not possible to randomly assign users to migrant and non-migrant conditions, nor was it feasible to control for all potential background variables on which these groups may differ. We conducted surveys with samples obtained through the online networks of two Indian nationals. 
Rather than trying to match migrant respondents with non-migrant respondents on an exhaustive list of background criteria, which would inevitably remain a limited one, sampling through one central network owner can help to ensure basic comparability of migrant and non-migrant respondent groups. Still, there are a number of variables, ranging from income levels to language skills to personality traits, that would be desirable to control for in future studies. In both studies, we could not detect any indication of systematic bias to our findings. For example, findings in Study 2 indicated that alternating SNS use was driving the size of the Facebook network, not the more established Orkut network where migrant and non-migrant users were not showing any differences. Success in Orkut socializing, in other words, was not a particular characteristic of those who went abroad.

To conclude, a farewell has to be said to Orkut as an Internet service. After ten years, the site has recently been shut down by its owner [45]. With next to no further technical development and overwhelming competition, it seems remarkable that Orkut was able to survive for so long. The theme of culture, migration and technology, however, is set to grow and intensify. As recent studies have shown, cross-cultural and transnational issues in technology use are gaining in prominence $[9,10,32,35]$. In some ways, it may be reassuring that users around the globe are not heading straight for a uniform, homogenous global village. This village will hold enough places to explore.

\section{Acknowledgments}

This research was partly funded by the EPSRC, UK and the ESRC, UK. The authors would like to thank Hriata Jahau and Karl D’Souza for their assistance in data collection.

\section{Author Contributions}

Authors contributed in equal parts to this work, with the first author leading on the data analysis.

\section{Conflicts of Interest}

The authors declare no conflict of interest.

\section{References}

1. Boyd, D.; Ellison, N.B. Social network sites: Definition, history, and scholarship. J. Comput. Mediat. Commun. 2008, 13, 210-230.

2. Haythornthwaite, C. Social networks and Internet connectivity effects. Inf. Community Soc. 2005, 8, 125-147.

3. Ledbetter, A.M. Patterns of media use and multiplexity: Associations with sex, geographic distance and friendship interdependence. New Media Soc. 2009, 11, 1187-1208.

4. Bernal, V. Eritrea on-line: Diaspora, cyberspace, and the public sphere. Am. Ethnol. 2005, 32, 660-675.

5. Burrell, J.; Anderson, K. "I have great desires to look beyond my world”: Trajectories of information and communication technology use among Ghanaians living abroad. New Media Soc. 2008, 10, 203-224. 
6. Castro, L.A.; Gonzalez, V.M. Being part of the life of one's hometown: Strategies to support community connectedness. PsychNol. J. 2008, 6, 61-82.

7. Hiller, H.H.; Franz, T.M. New ties, old ties and lost ties: The use of the Internet in diaspora. New Media Soc. 2004, 6, 731-752.

8. Komito, L. Social media and migration: Virtual community 2.0. J. Am. Soc. Inf. Sci. Technol. 2011, 62, 1075-1086.

9. Shklovski, I.; Vertesi, J.; Lindtner, S. Introduction to this special issue on transnational HCI. Hum. Comput. Interact. 2014, 29, 1-21.

10. Qiu, L.; Lin, H.; Leung, A.K. Cultural differences and switching of in-group sharing behavior between an American (Facebook) and a Chinese (Renren) social networking site. J. Cross-Cult. Psychol. 2013, 44, 106-121.

11. Ellison, N.B.; Steinfield, C.; Lampe, C. The benefits of Facebook “friends": Social capital and college students' use of online social network sites. J. Comput. Mediat. Commun. 2007, 12, 1143-1168.

12. Ellison, N.B.; Steinfield, C.; Lampe, C. Connection strategies: Social capital implications of Facebook-enabled communication practices. New Media Soc. 2011, 13, 873-892.

13. Wilson, R.E.; Gosling, S.D.; Graham, L.T. A review of Facebook research in the social sciences. Perspect. Psychol. Sci. 2012, 7, 203-220.

14. Burke, K.; Kraut, R.; Marlow, C. Social capital on Facebook: Differentiating uses and users. In Proceedings of the SIGCHI, Vancouver, BC, Canada, 7-12 May 2011; pp. 571-580.

15. Tong, S.T.; van der Heide, B.; Langwell, L.; Walther, J.B. Too much of a good thing? The relationship between number of friends and interpersonal impressions on Facebook. J. Comput. Mediat. Commun. 2009, 13, 531-549.

16. Kuss, D.J.; Griffiths, M.D. Online social networking and addiction-A review of the psychological literature. Int. J. Environ. Res. Public Health 2011, 8, 3528-3552.

17. Eastin, M.S.; LaRose, R. Alt.support: Modeling social support online. Comput. Hum. Behav. 2005, 21, 977-992.

18. Kalpidou, M.; Costin, D.; Morris, J. The relationship between Facebook and the well-being of undergraduate college students. Cyberpsychol. Behav. Soc. Netw. 2011, 14, 183-189.

19. Vergeer, M.; Pelzer, B. Consequences of media and Internet use for offline and online network capital and well-being. A causal model approach. J. Comput. Mediat. Commun. 2009, 15, 189-210.

20. Granovetter, M.S. The strength of weak ties. Am. J. Sociol. 1973, 78, 1360-1380.

21. Binder, J.F.; Roberts, S.G.; Sutcliffe, A.G. Closeness, loneliness, support: Core ties and significant ties in personal communities. Soc. Netw. 2012, 34, 206-214.

22. Donath, J.; Boyd, D. Public displays of connection. BT Technol. J. 2004, 22, 71-82.

23. Manago, A.M.; Taylor, T.; Greenfield, P.M. Me and my 400 friends: The anatomy of college students' Facebook networks, their communication patterns, and well-being. Dev. Psychol. 2012, 48, 369-380.

24. Lampe, C.; Ellison, N.; Steinfield, C. A familiar Face(book): Profile elements as signals in an online social network. In Proceedings of the SIGCHI Conference on Human Factors in Computing Systems, San Jose, CA, USA, 28 April-3 May 2007; pp. 435-444. 
25. Lewis, K.; Kaufman, J.; Gonzalez, M.; Wimmer, A.; Christakis, N. Tastes, ties, and time: A new social network dataset using Facebook.com. Soc. Netw. 2008, 30, 330-342.

26. Burke, M.; Marlow, C.; Lento, T. Feed me: Motivating newcomer contribution in social network sites. In Proceedings of the SIGCHI, Boston, MA, USA, 4-9 April 2009; pp. 945-954.

27. Binder, J.F.; Howes, A.; Sutcliffe, A.G. The problem of conflicting social spheres: Effects of network structure on experienced tension in social network sites. In Proceedings of the SIGCHI, Boston, MA, USA, 4-9 April 2009; pp. 965-974.

28. Binder, J.F.; Howes, A.; Smart, D. Harmony and tension on social network sites: Side-effects of increasing online interconnectivity. Inf. Commun. Soc. 2012, 15, 1279-1297.

29. Hargittai, E. Whose space? Differences among users and non-users of social network sites. J. Comput. Mediat. Commun. 2007, 13, 276-297.

30. Skeels, M.M.; Grudin, J. When social networks cross boundaries: A case study of workplace use of Facebook and LinkedIn. In Proceedings of the GROUP'09, Sanibel Island, FL, USA, 10-13 May 2009; pp. 95-104.

31. Berry, J.W. Immigration, acculturation and adaptation. Appl. Psychol. 1997, 46, 5-68.

32. Lazzari, $M$. The role of social networking services to shape the double virtual citizenship of young immigrants in Italy. In Proceedings of the IADIS International Conference ICT, Society and Human Beings, Lisbon, Portugal, 21-23 July 2012; pp. 11-18.

33. Jasinskaja-Lahti, I.; Liebkind, K.; Jaakkola, M.; Reuter, A. Perceived discrimination, social support networks, and psychological well-being among three immigrant groups. J. Cross-Cult. Psychol. 2006, 37, 293-311.

34. Chen, W. Internet-usage patterns of immigrant in the process of intercultural adaptation. Cyberpsychol. Behav. Soc. Netw. 2010, 13, 387-399.

35. Lee, E.J.; Lee, L.; Jang, J. Internet for the internationals: Effects of Internet use motivations on international students’ college adjustment. Cyberpsychol. Behav. Soc. Netw. 2011, 14, 433-437.

36. Hendrickson, B.; Rosen, D.; Aune, R.K. An analysis of friendship networks, social connectedness, homesickness, and satisfaction levels of international students. Int. J. Intercult. Relat. 2011, 35, 281-295.

37. Smith, R.A.; Khawaja, N.G. A review of the acculturation experiences of international students. Int. J. Intercult. Relat. 2011, 35, 699-713.

38. Jung, T.; Youn, H.; McClung, S. Motivations and self-presentation strategies on Korean-based “Cyworld” weblog format personal homepages. Cyberpsychol. Behav. 2007, 10, 24-31.

39. Tokunaga, R.S. Friend me or you'll strain us: Understanding negative events that occur over social networking sites. Cyberpsychol. Behav. Soc. Netw. 2011, 14, 425-432.

40. Thelwall, M. Homophily in MySpace. J. Am. Soc. Inf. Sci. Technol. 2009, 60, 219-231.

41. Abbas, S.M.A. Homophily, popularity and randomness: Modelling growth of online social network. In Proceedings of the 2013 International Conference on Autonomous Agents and Multi-Agent Systems (AAMAS), Saint Paul, MN, USA, 6-10 May 2013; pp. 135-142.

42. Boyd, D. Friends, Friendsters, and MySpace Top 8: Writing Community into Being on Social Network Sites. Available online: http://firstmonday.org/ojs/index.php/fm/article/view/1418/1336 (accessed on 1 June 2014).

43. Buys, C.J.; Larson, K.L. Human sympathy groups. Psychol. Rep. 1979, 45, 547-553. 
44. Dunbar, R.I.M.; Spoors, M. Social networks, support cliques, and kinship. Hum. Nat. 1995, 6, 273-290.

45. CNET News. Google to Kill Social Network Orkut. Available online: http://www.cnet.com/uk/ news/google-to-kill-social-network-orkut/ (accessed on 1 November 2014).

(C) 2014 by the authors; licensee MDPI, Basel, Switzerland. This article is an open access article distributed under the terms and conditions of the Creative Commons Attribution license (http://creativecommons.org/licenses/by/4.0/). 